\title{
Molecular Crosstalk Between PAMP-Triggered Immunity and Photosynthesis
}

\author{
Vera Göhre, ${ }^{1}$ Alexandra M. E. Jones, ${ }^{2}$ Jan Sklenář, ${ }^{2}$ Silke Robatzek, ${ }^{2}$ and Andreas P. M. Weber ${ }^{3}$ \\ ${ }^{1}$ Heinrich-Heine University, Institute for Microbiology, Universitätsstr. 1, 40225 Düsseldorf, Germany; ${ }^{2}$ The Sainsbury \\ Laboratory, Norwich Research Park, Norwich NR4 7UH, U.K.; ${ }^{3}$ Heinrich-Heine University, Institute for Plant Biochemistry, \\ Universitätsstr. 1, 40225 Düsseldorf, Germany
}

Submitted 24 November 2011. Accepted 8 April 2012.

\begin{abstract}
The innate immune system allows plants to respond to potential pathogens in an appropriate manner while minimizing damage and energy costs. Photosynthesis provides a sustained energy supply and, therefore, has to be integrated into the defense against pathogens. Although changes in photosynthetic activity during infection have been described, a detailed and conclusive characterization is lacking. Here, we addressed whether activation of early defense responses by pathogen-associated molecular patterns (PAMPs) triggers changes in photosynthesis. Using proteomics and chlorophyll fluorescence measurements, we show that activation of defense by PAMPs leads to a rapid decrease in nonphotochemical quenching (NPQ). Conversely, NPQ also influences several responses of PAMP-triggered immunity. In a mutant impaired in NPQ, apoplastic reactive oxygen species production is enhanced and defense gene expression is differentially affected. Although induction of the early defense markers WRKY22 and WRKY29 is enhanced, induction of the late markers $P R 1$ and $P R 5$ is completely abolished. We propose that regulation of NPQ is an intrinsic component of the plant's defense program.
\end{abstract}

Plants sense potential pathogens by recognizing conserved pathogen-associated molecular patterns (PAMPs). Well-characterized PAMPs are flg22, a peptide derived from the bacterial motor protein flagellin; elf18, a peptide from bacterial elongation factor (EF)-Tu; and chitin, a component of the fungal cell wall (Kunze et al. 2004; Wan et al. 2008). The PAMP flg22 is recognized by the receptor flagellin sensing 2 (FLS2) (Gomez-Gomez and Boller 2000). Upon binding, the receptor dimerizes with brassinolide-insensitive 1-associated kinase (BAK1), activating a set of defense responses. These involve production of reactive oxygen species (ROS) in the apoplast mediated by the NAPDH oxidase respiratory burst homolog (RbohD), global changes in gene expression (e.g., WRKY transcription factors and pathogenesis-related $[P R]$ genes), production of defense compounds, and stomatal closure, which collectively contribute to PAMP-triggered immunity (PTI). Long-term exposure to flg22 induces a growth inhibition in seedlings (Gomez-Gomez et al. 1999). Likewise, elf18 and chitin elicit

S. Robatzek and A. P. M. Weber contributed equally.

Corresponding author: V. Göhre;

E-mail: Vera.Goehre@uni-duesseldorf.de

* The $\boldsymbol{e}$-Xtra logo stands for "electronic extra" and indicates that one supplementary figure and one supplementary table are published online. defense responses and, thereby, trigger PTI (Kunze et al. 2004; Wan et al. 2008). In most cases, PTI efficiently prevents pathogens from infecting the plant without any visible symptoms. To overcome this level of immunity, virulent and avirulent pathogens inject effector proteins into the plant cell, which interfere with defense responses to suppress immunity and gain excess to photo-assimilates. In response to effectors, plants have evolved resistance $(\mathrm{R})$ proteins that recognize specifically individual effectors and activate effector-triggered immunity (ETI) (Jones and Dangl 2006). Defense responses are potentiated, leading to programmed cell death of the hypersensitive response (HR) type. This rapid, localized cell death involves a burst of ROS in the chloroplast (Zurbriggen et al. 2009) and inhibits spread of the pathogen.

There are obvious reasons why contact with pathogens has an impact on photosynthesis. Induction of defense responses requires energy (Swarbrick et al. 2006), and photo-assimilates serve as a carbon source in the synthesis of defense compounds (Hammerschmidt 1999). This causes an increased demand for photosynthesis in the plant (Berger et al. 2007b). In addition, in a virulent interaction, pathogens withdraw sugars and, thereby, further augment the consumption of carbon compounds. For example, fungal and bacterial pathogens target host sugar transporters of the SWEET family to promote sugar efflux from the host cell (Chen et al. 2010) and induce sink genes (e.g., a cellwall invertase in an infected source leaf) (Berger et al. 2004). In contrast to the expected increase, infection with virulent or avirulent pathogens often leads to a decrease in photosynthesis, as shown by chlorophyll fluorescence measurements (Barriuso et al. 2008; Berger et al. 2007b; Ishiga et al. 2009; RodriguezMoreno et al. 2008) and proteomics studies (Afroz et al. 2009; Diaz-Vivancos et al. 2008; Ji et al. 2009; Marsh et al. 2010; Pineda et al. 2010; Yang et al. 2010; Zhou et al. 2006). The decrease in photosynthesis in response to infection might be an active process as part of the plant defense program to limit carbon source availability for the pathogen or result from a prioritization of metabolic processes in favor of defense reaction (e.g., sustaining the high NADPH demand of RbohD activity during the ROS burst) (Bolton 2009).

To date, most studies investigating the link between immunity and photosynthesis have been carried out using inoculated plants. To reduce complexity of the interaction between plants and pathogens, individual PAMPs can be used, which trigger defined and quantifiable defense responses. In this study, flg22 perception in cell culture and in plants was used to investigate changes in the chloroplast proteome and in photosynthesis during the activation of PTI. Changes in the proteome were marginal and involved, in total, only six photosynthetic proteins. PsbS, a subunit of photosystem II that plays a role in en- 
ergy dissipation to protect the photosystems against damage by excess light (nonphotochemical quenching [NPQ]), is rapidly downregulated after flg22 treatment. A corresponding decrease in NPQ was observed upon PAMP treatment. Conversely, in a mutant impaired in NPQ, lacking PsbS and the violaxanthin de-epoxidase Vde1 (called $p s b S$ vdel here to refer to the proteins, more commonly known as $n p q 4 n p q 1$ ), PAMP responses are altered, suggesting a link between the protection of photosystems by NPQ and flg22 signaling.

\section{RESULTS}

\section{Photosynthetic proteins are affected during PTI.}

To investigate flg22-induced changes in photosynthesis at the protein level, quantitative mass spectrometric analyses of enriched organelle fractions was performed. A green cell culture derived from the ecotype Landsberg erecta was metabolically labeled with the stable isotope ${ }^{15} \mathrm{~N}$ or unlabeled with naturally occurring ${ }^{14} \mathrm{~N}$ (abundance of the heavy isotope is $0.364 \%$ ) for ratiometric quantification between untreated samples and those treated with $100 \mathrm{nM}$ flg22. Two time points in four replicates, including reciprocal labeling, were sampled after elicitation with flg22: $30 \mathrm{~min}$, to monitor protein degradation processes, or $2 \mathrm{~h}$, which also allows protein synthesis to take place. Using cell culture offers several advantages: cells can easily be labeled with ${ }^{15} \mathrm{~N}$ to $>95 \%$ as is required for quantification, the cells are identical as opposed to differentiated cell types in plants, and flg22 treatment leads to homogenous stimulation of all cells within seconds. Chloroplasts were enriched using a fast protocol to minimize changes occurring during isolation but at the cost of contamination with other organelles. From gel-liquid chromatography (LC) analysis of enriched chloroplast fractions, 1,923 proteins could be identified. A fourth of these are localized in chloroplasts based on the AT_CHLORO database (Ferro et al. 2010), confirming the expected contamination of the fraction (Supplementary Table S1). Of the identified proteins, 62 are involved in photosynthesis or carbon fixation.
Ratiometric quantification requires more peptide hits for each protein in both metabolic states. In all, 1,022 of the 1,923 identified proteins could also be quantified both $30 \mathrm{~min}$ and $2 \mathrm{~h}$ after flg 22 treatment. Of these quantified proteins, 314 are predicted to be localized to the chloroplast. They function in different processes: 40 are involved in photosynthesis and carbon fixation, which covers one-third of the proteins annotated as involved in these processes; and the others have proposed functions in metabolism of amino acids, lipids, vitamins, and pigments, as well as chloroplast gene expression system and chloroplast transporters.

Only marginal changes occurred after flg22 treatment: twofold changes, which are frequently used as a cut-off in similar studies, were rare. To detect minor but significant changes to the proteome, we conducted a rank product analysis on the dataset, a nonparametric test that can deal with noisy and variable data and is very sensitive if the data are strongly reproducible (Breitling et al. 2004). We considered only proteins present in at least three biological replicates that did not change more than $7 \%$ in the controls. Of the 314 quantified chloroplast proteins, 18 were differentially regulated after $30 \mathrm{~min}$ and 17 after $2 \mathrm{~h}$ flg22 treatment (Table 1), nine of which occurred in both time points. These differentially regulated proteins comprise the following photosynthetic proteins: at both time points, $\mathrm{RbcL}$ (large subunit of the ribulose-bisphosphate carboxylase), AtpA (ATP synthase CF1 $\alpha$ chain), and PsbS (photosystem II 22$\mathrm{kDa}$ chloroplast protein); and, additionally at $2 \mathrm{~h}, L H C B 4$ (chlorophyll A-B binding protein is altered).

At the RNA level, microarray data show that most photosynthetic genes are downregulated (Denoux et al. 2008), which we confirmed for RBCL, PSBS, ATPA, and LHCB4 by quantitative reverse-transcriptase polymerase chain reaction (qRT-PCR). Downregulation of $R B C L$ and $P S B S$ supports the results from our proteomics study (Fig. 1). However, ATPA and LHCB4 are downregulated at the RNA level but the two proteins accumulate upon flg22 treatment. Such an opposite behavior comparing protein and RNA levels emphasizes the importance of simultaneously quantifying the proteome in addition to the transcriptome.

Table 1. Chloroplast proteins are significantly changed after flg 22 treatment ${ }^{\mathrm{a}}$

\begin{tabular}{|c|c|c|c|}
\hline AGI & $30 \mathrm{~min}$ & $2 \mathrm{~h}$ & Protein \\
\hline AT1G07660.1 & ns & 0.91 & Histone H4 \\
\hline AT1G14810.1 & ns & 0.94 & Semialdehyde dehydrogenase family protein \\
\hline AT1G15690.1 & ns & 0.94 & AVP1 (vacuolar-type H+-pumping pyrophosphatase 1) \\
\hline AT1G44575.1 & 0.93 & 0.93 & PsbS/NPQ4 (nonphotochemical quenching) \\
\hline AT1G55490.1 & 1.06 & 0.92 & CPN60B (chaperonin 60 beta) \\
\hline AT1G78380.1 & 1.04 & ns & GSTU19 (glutathione transferase 8) \\
\hline AT2G07698.1 & 0.97 & ns & ATP synthase $\alpha$ chain \\
\hline AT2G15620.1 & 0.92 & ns & NIR1 (nitrite reductase) \\
\hline AT2G21170.1 & ns & 0.89 & TIM (triosephosphate isomerase, chloroplastic) \\
\hline AT2G36460.1 & 1.06 & ns & Fructose-bisphosphate aldolase, putative \\
\hline AT3G07770.1 & 0.96 & ns & ATP binding, putative \\
\hline AT3G13470.1 & 0.92 & 0.93 & Chaperonin, putative \\
\hline AT3G47520.1 & 1.00 & 0.95 & MDH (malate dehydrogenase) \\
\hline AT3G52930.1 & 1.15 & ns & Fructose-bisphosphate aldolase, putative \\
\hline AT4G02510.1 & 1.03 & ns & TOC159 (plastid protein import 2) \\
\hline AT4G34200.1 & 0.86 & 0.93 & EDA9 (embryo sac development arrest 9) \\
\hline AT5G01530.1 & ns & 1.11 & LHCB4 (chlorophyll A-B binding protein CP29) \\
\hline AT5G09650.1 & 1.04 & ns & PPA6 (pyrophosphorylase 6) \\
\hline AT5G11880.1 & 1.06 & ns & Diaminopimelate decarboxylase, putative \\
\hline AT5G13490.1 & 0.97 & 0.95 & AAC2 (ADP/ATP carrier 2) \\
\hline AT5G20720.1 & 0.89 & 0.90 & CPN20 (chaperonin 20) \\
\hline AT5G23060.1 & ns & 0.89 & $\mathrm{CaS}$ (calcium sensing receptor) \\
\hline AT5G26742.1 & ns & 1.15 & EMB1138 (embryo defective 1138) \\
\hline AT5G50920.1 & ns & 1.04 & CLPC (heat shock protein $93-\mathrm{V}$ ) \\
\hline AtCg00120.1 & 1.05 & 1.01 & ATP synthase \\
\hline AtCg00490.1 & 0.92 & 0.94 & RbcL (Ribulose bisphosphate carboxylase large chain) \\
\hline
\end{tabular}

${ }^{a}$ Ratio of treated/untreated samples $30 \mathrm{~min}$ and $2 \mathrm{~h}$ after flg22 treatment is given for proteins that were significantly affected based on a rank product analysis. Bold indicates proteins affected in light or dark reaction of photosynthesis, $n s$ : no significant change in this time point. 
NPQ is affected by treatment with PAMPs.

Based on our data, PsbS was one of the few photosynthetic proteins that rapidly decreased in abundance after flg 22 treatment; this suggested that NPQ might be reduced upon flg22 treatment. NPQ can be quantified by chlorophyll fluorescence measurements. To determine the influence of flg22 treatment on NPQ, we decided to use seedlings grown in liquid media, which are the same conditions used for seedling growth inhibi-

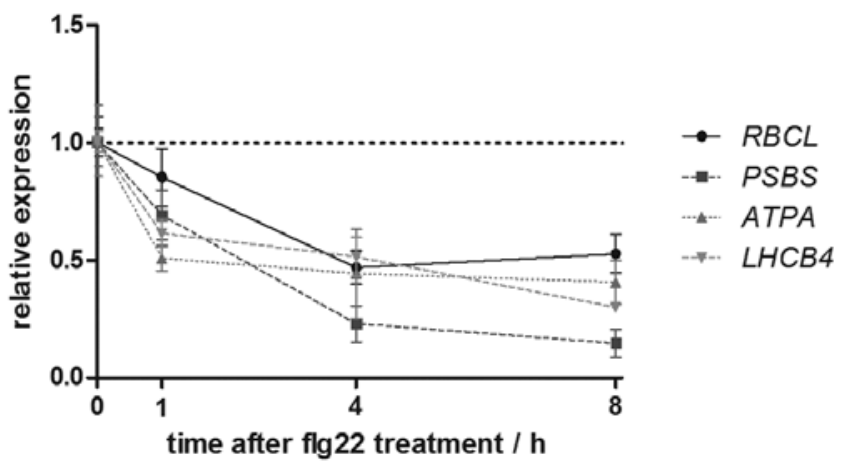

Fig. 1. Photosynthetic genes are rapidly regulated upon flg22 perception. Gene expression for the candidate genes RBCL, PSBS, ATPA, and LHCB4 was measured by quantitative reverse-transcriptase polymerase chain reaction in 2-week-old seedlings treated with $10 \mu \mathrm{M}$ flg22 for the indicated time. Expression was normalized to actin levels and to untreated conditions. At the RNA level, all photosynthetic genes are downregulated, even ATPA and LHCB4 that showed an increase in the proteomics dataset. Three independent experiments gave similar results.
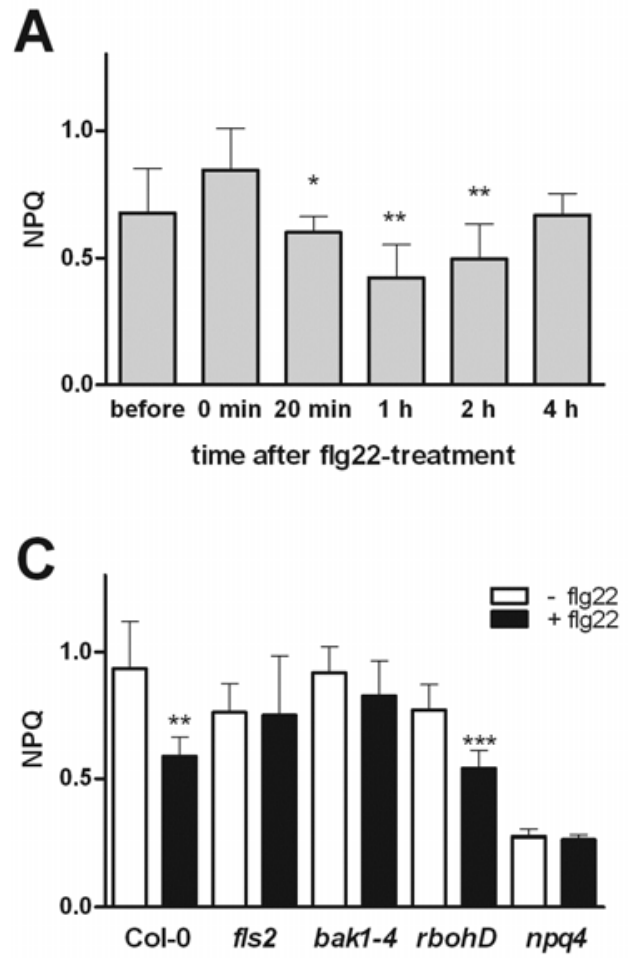

tion assays in a previous study (Gomez-Gomez et al. 1999). These conditions allow PAMPs to rapidly diffuse and get in contact with the plant tissues without wounding of the leaf material, which occurs during infiltration. The chlorophyll fluorescence of submerged seedlings differs from untreated seedlings: Fv/Fm ranges between 0.6 and 0.7 compared with 0.83 in adult leaves. Nevertheless, under these conditions optimized for uniform PAMP responsiveness, relative changes can be reliably measured.

First, changes in NPQ triggered by flg22 treatment were measured over time. Treatment with $10 \mu \mathrm{M}$ flg22 in the light led to a decrease in NPQ as early as 20 min (Fig. 2A). At $1 \mathrm{~h}$, NPQ reached a minimum and then recovered, probably reflecting adaptation processes to the stress imposed by flg22 responses. Defense responses such as flg22-triggered ROS production are dose dependent (Felix et al. 1999). Therefore, we measured NPQ reduction $1 \mathrm{~h}$ after treatment with varying flg22 concentrations in the media. At $100 \mathrm{nM}$ flg22, a significant reduction in NPQ was observed, and NPQ further decreased with rising flg22 concentration (Fig. 2B), confirming that the response of NPQ to flg22 is dose-dependent. At $10 \mu \mathrm{M}$ flg22, the difference was maximal; therefore, all further experiments were carried out at this concentration, and NPQ was measured $1 \mathrm{~h}$ after treatment.

The FLS2 receptor binds flg22 directly and this interaction is required to trigger defense responses (Chinchilla et al. 2006). Therefore, we tested the hypothesis that flg22-induced changes in NPQ are mediated by the FLS2 receptor and its signaling partner, BAK1 (Chinchilla et al. 2007). In neither the receptor mutant $f l s 2$ nor bakl-4 were changes in NPQ observed
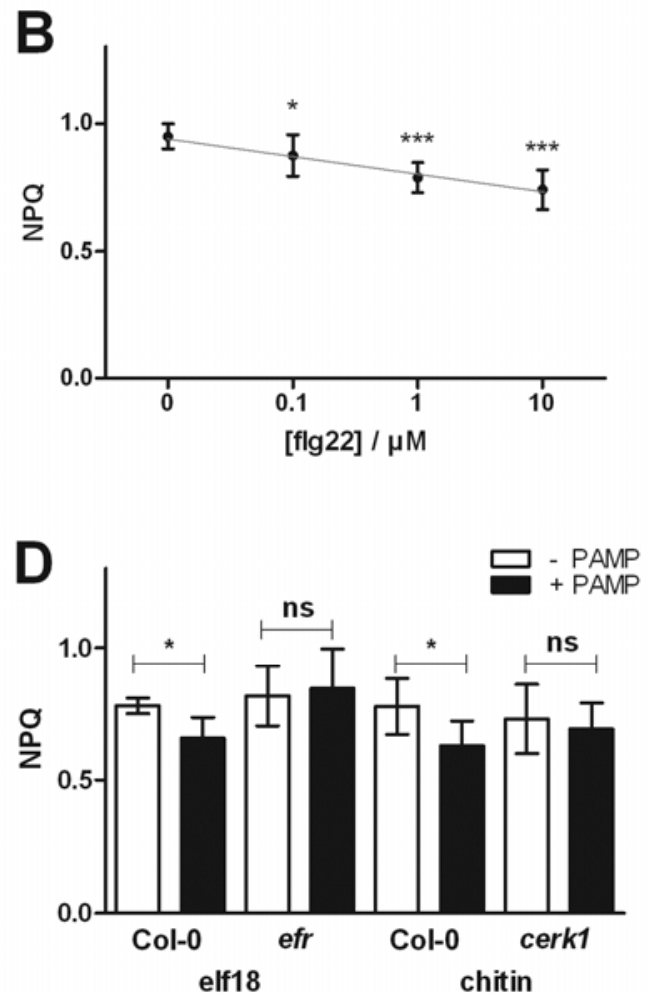

Fig. 2. Pathogen-associated molecular pattern (PAMP) perception triggers a decrease in nonphotochemical quenching (NPQ). NPQ was measured by chlorophyll fluorescence in light-adapted seedlings. A, Treatment with flg22 causes a time-dependent decrease in NPQ, which reaches a minimum at $1 \mathrm{~h}$ and then recovers despite the constant presence of flg22. B, Dose-response curve of flg22-induced changes in NPQ $1 \mathrm{~h}$ after flg22 addition; $1 \mu \mathrm{M}$ flg22 was used in all experiments. C, The FLS2 receptor and its signaling partner BAK1 are required for the decrease in NPQ. The flg22-triggered reactive oxygen species burst is not required for reduction in NPQ, because the rbohD mutant showed a reduced NPQ. D, Other PAMPs such as elf18 and chitin cause a decrease in NPQ in the wild type but not in the respective receptor mutants. Values are given as mean \pm standard deviation, $n \geq 6$. Asterisks indicate significant differences compared with untreated samples based on Student's $t$ test; *, **, and *** indicate $P<0.05,0.01$, and 0.001 , respectively; ns $=$ not significant. Several independent experiments gave similar results. 
(Fig. 2C). To further test whether PsbS is necessary for the flg22-induced reduction in NPQ, the $p s b S$ vde1 double mutant lacking PsbS (Li et al. 2000) and the violaxanthin de-epoxidase Vde1 involved in energy dissipation via the xanthophyll cycle (Niyogi et al. 1998) was treated with flg22. NPQ was already lowered in this mutant compared with the wild type, and treatment with flg22 did not further decrease NPQ (Fig. 2C). Next, we tested whether NPQ decreases in the wild type specifically in response to flg22 or if it also responds to other PAMPs, such as elf18 and chitin. As expected, treatment with these PAMPs leads to a reduction in NPQ similar to flg22 treatment (Fig. 2D). Hence, activation of PTI seems to have an impact on the chloroplast, and changes in NPQ are a measurable output of these early changes.

Downregulation of NPQ in response to flg22 requires the same plasma-membrane-localized signal perception as defense signaling (FLS2 and BAK1) and probably involves some of the known cytosolic signaling components. However, NPQ takes place in the chloroplast, which is highly redox regulated. To exclude the possibility that the flg22-triggered oxidative burst in the apoplast is required for the observed decrease in NPQ via a receptor-independent redox-link, we used the rbohD mutant, where the PAMP-responsive oxidative burst is lost (Torres et al. 2002). NPQ still decreased in response to
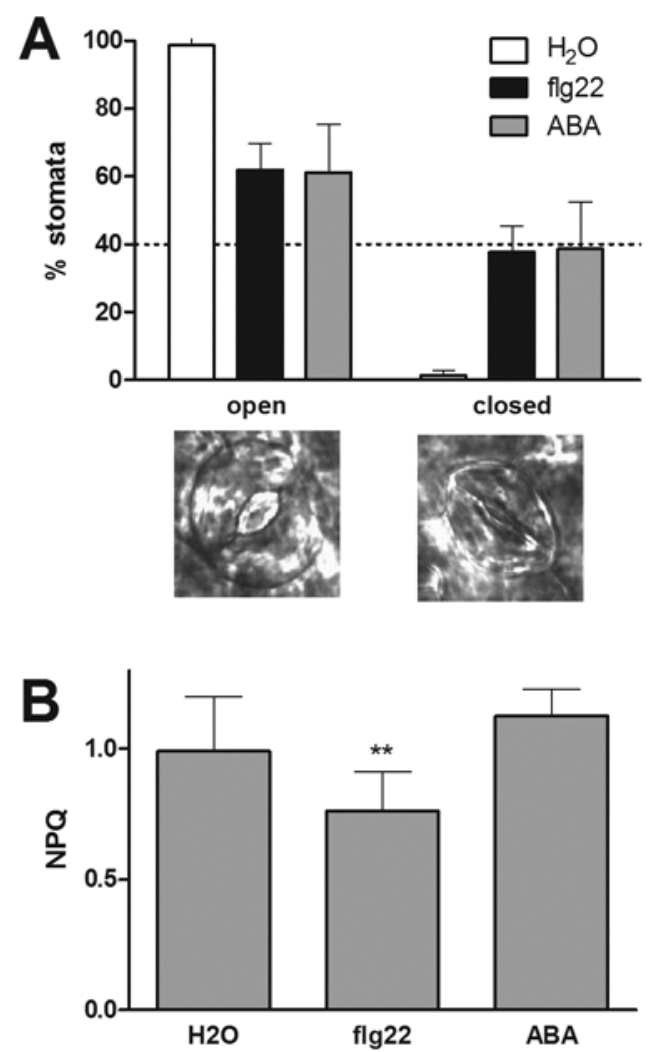

Fig. 3. Stomatal closure is not the cause for the flg22-triggered decrease in nonphotochemical quenching (NPQ). A, NPQ was measured by chlorophyll fluorescence in light-adapted seedlings. abscissic acid (ABA) does not induce changes in NPQ that are observed with flg22. Values are given as mean \pm standard deviation (SD), $n \geq 6$. Asterisks indicate significant differences compared with untreated samples based on Student's $t$ test; *, **, and *** indicate $P<0.05,0.01$, and 0.001 , respectively. B, Stomata of seedlings grown under identical conditions were observed under the microscope and aperture was measured for 100 stomata each. Values are given \pm SD (upper panel) and representative picture for open and closed stomata is shown (lower panel). Under these growth conditions $\left(\mathrm{H}_{2} \mathrm{O}\right)$, almost all stomata are open. ABA and flg22 treatment both induce stomatal closure of approximately $40 \%$ of the stomata, suggesting that stomatal closure does not cause changes in NPQ. Three independent experiments gave similar results. flg22 treatment in the $r b o h D$ mutant (Fig. 2C). This indicates that the apoplastic redox changes do not influence the chloroplast. Therefore, other signaling pathways downstream of the receptor such as cytosolic redox, calcium or $\mathrm{pH}$ changes, mitogen-activated protein kinases (MAPK), calcium-dependent protein kinases (CDPK), or hormones might relay the signal independently of extracellular responses.

Alternatively, changes in NPQ induced by flg22 might not be cell-autonomous. As an early defense response, flg22 triggers stomatal closure (Melotto et al. 2006), which reduces the entry of bacterial pathogens into the leaf tissue. Stomatal closure can also alter gas exchange and, thereby, affect photosynthesis and NPQ. To test whether stomatal closure triggers the observed changes in NPQ, seedlings were treated with abscissic acid (ABA), which induces closure in response to drought. In the submerged seedlings, both flg22 and ABA induced approximately $40 \%$ of the stomata to close (Fig. 3A). However, ABA did not induce a decrease in NPQ (Fig. 3B). Therefore, the observed changes in NPQ upon flg22 treatment are not induced by altered gas exchange.

\section{Altered photosynthesis contributes \\ to seedling growth inhibition.}

In addition to rapid induction of defense responses, longterm exposure to flg22 causes growth inhibition in seedlings (Gomez-Gomez et al. 1999). Partly, this can be explained by a stabilization of DELLA proteins by flg22 which, in turn, represses gibberellin signaling and, hence, growth (Navarro et al. 2008). However, additional factors (e.g., energy status) altered by flg 22 could also contribute to the seedling growth inhibition. Therefore, we measured photosynthetic activity during the growth inhibition response by chlorophyll fluorescence. Seedlings grown for 7 days in the presence of flg22 show a concentration-dependent growth arrest, which correlates with a decrease in the proportion of absorbed energy used for photosynthesis in light-adapted (QY) and dark-adapted (QY_max) seedlings (Fig. 4). This decrease in use of energy for photosynthesis is complemented by an increase in NPQ, probably to release the excess energy. This increase in NPQ during longterm exposure is opposite to the initial decrease immediately after flg22 treatment and probably reflects different mechanisms acting during activation of defense and adaptation to prolonged PAMP exposure. In conclusion, long-term flg22 treatment decreases electron transfer through photosystem II, which limits energy production and, thereby, might retard growth.

\section{Mutants impaired in NPQ show altered flg22 responses.}

Perception of flg22 influences NPQ by reducing the amount of PsbS. To test the converse (i.e., whether NPQ influences flg22 signaling), $p b s S, v d e 1$, and $p s b S$ vdel lacking NPQ were examined for flg22 responsiveness. Already, in the single mutants, the apoplastic ROS burst mediated by RbohD was enhanced, and this was more pronounced in the double mutant (Fig. 5A). This could be due to altered FLS2 levels but, in the psbS vde1 mutant, induction of FLS2 expression is unaltered (Fig. 5B). Downstream flg22 signaling leads to transcriptional induction of the transcription factors WRKY22 and WRKY29 (Asai et al. 2002). Similar to the ROS burst, expression of these transcription factors was slightly enhanced in the $p s b S$ vde1 mutant (Fig. 5C). Together with the observation that flg22 leads to decreased NPQ upon short-term treatments, these data suggest that NPQ might counteract some of the flg22-induced defense responses. On the other hand, induction of the late flg22-responsive genes $P R 1$ and $P R 5$ is completely abolished in $p s b S$ vdel (Fig. 5C) and, in the single mutants, with the exception of PR5, induction is not completely abolished but strongly reduced in $p s b S$ (Supplementary Fig. S1). In 
contrast to the observed changes in $P R$ gene expression, the seedling growth inhibition is unaltered (Fig. 5D). Therefore, changes in flg22 responses occurring within minutes and hours such as ROS burst and defense gene expression seem to correlate with the flg22-induced decrease in NPQ (Fig. 2), whereas long-term exposure to flg22 for days seems to cause adaptation processes in photosynthesis complemented by an increase in NPQ correlating with the seedling growth inhibition (Fig. 4). These data imply that the level of NPQ exerted by the chloroplast influences flg22 signaling and might hint at a feedback regulation from the chloroplast to PTI.

\section{DISCUSSION}

\section{Activation of PTI influences photosynthesis.}

It is well known that the chloroplast plays an important role in ETI. Upon recognition of effectors by R proteins, an oxidative burst is produced in the chloroplast leading to HR-type programmed cell death (Zurbriggen et al. 2009). In this study, we have investigated the link between the chloroplast and PTI. We show that perception of PAMPs leads to alterations in photosynthetic parameters.

To determine changes in photosynthetic proteins during activation of PTI, we carried out a quantitative proteomics approach on chloroplasts isolated from cell cultures. In addition to the obvious advantage of cell culture (homogenous and rapid elicitation of identical cells), we also noticed limitations. Enriched chloroplasts resembled proplastids rather than fully developed chloroplasts. Even though a green cell culture was used, which is considered to be photosynthetically active, abundance of photosynthetic proteins as well as chlorophyll levels were low compared with levels expected in fully active chloroplasts. This might explain why not all photosynthetic proteins could be quantified and the observed changes were small, even though their high abundance in chloroplasts can hamper quantitative proteomics of other chloroplast processes (Cellar et al. 2008). Nevertheless, we identified four photosynthetic proteins which are altered by flg22 perception: LhcB4 and AtpA accumulate upon flg22 treatment, while PsbS and RbcL levels decrease. Decrease in $\mathrm{RbcL}$, the rate-limiting enzyme of $\mathrm{CO}_{2}$ fixation, can explain the previously described reduction in $\mathrm{CO}_{2}$ fixation after flg22 treatment (Freeman and Beattie 2009). Induction of LhcB4 suggests higher energy input into photosystem II. Concomitant reduction in PsbS levels might lead to an increased production of ROS at photosystem II if the energy cannot be released via the photosynthetic chain or NPQ. In addition, the level of the ATP synthase increases, which might play a role in downregulation of NPQ because, in mutants with decreased levels of ATP synthase, NPQ increases (Bosco et al. 2004). Overall, the changes are only marginal, so that they probably do not alter the photosynthetic yield. We suggest that a model PAMP perception might prime the chloroplast to respond with a rapid burst of ROS in case the infection is successful and effectors are perceived.

\section{PAMP perception differentially influences NPQ.}

In addition to changes in enzymes directly involved in the electron flow or carbon fixation, accumulation of the photosystem II subunit PsbS is decreased by flg22 treatment for $30 \mathrm{~min}$ or $2 \mathrm{~h}$. PsbS protects photosystem II against excess light energy by NPQ. Decrease at the protein level was reflected in a reduction in NPQ. NPQ drops as early as 20 min after PAMP perception, reaches a minimum at 1 to $2 \mathrm{~h}$, and then recovers. This timing correlates well with the induction of early and late defense responses. At longer, 7-day exposures to flg22, NPQ increases with a concomitant decrease in the use of energy for photosynthesis QY; this correlates with seedling growth inhibition. We suggest that reduced energy use efficiency in the presence of flg 22 might be one of the causes of retarded growth of the seedlings.

In their natural environment, plants are constantly exposed to microbes and, thus, perceive PAMPs continually. Such a prolonged exposure to PAMPs would lead to a significant yield loss if photosynthetic activity is constantly reduced. However, this is not observed. In infections with bacterial pathogens, plants respond to mixtures of PAMPs, so that balancing effects of different PAMPs in dosage and timing (Aslam et al. 2009) might influence the extent of growth inhibition. NPQ is increased during infection with virulent (Berger et al. 2007a) and avirulent (Bonfig et al. 2006) pathogens. During investigation of fluorescence parameters at early time points, 6 to $9 \mathrm{~h}$ after infection with virulent bacteria, Matous and associates (2006)

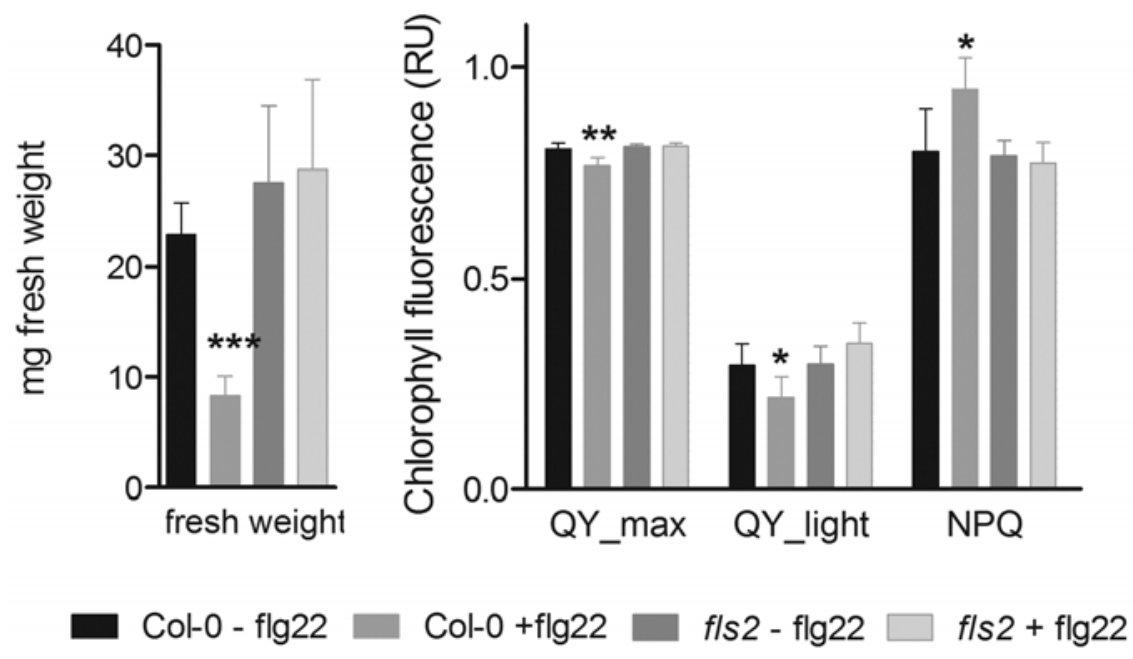

Fig. 4. Long-term adaptation to flg22 involves changes in photosynthesis and nonphotochemical quenching (NPQ). Wild-type (Col-0) and receptor mutant (fls2) seedlings were grown for 7 days in the presence of flg22. Fresh weight was measured for treated and untreated seedlings after this period. The described growth inhibition phenotype was observed in the wild type but not in the receptor mutant (Gomez-Gomez et al. 1999). Of the same seedlings, quantum yield (i.e., the use of energy for photosynthesis in light-adapted [QY] and dark-adapted [QY_max] seedlings) and NPQ was measured by chlorophyll fluorescence. In Col-0, QY and QY-max decrease upon flg22 treatment, complemented by an increase in NPQ. In $f l s 2$, no changes were observed. Values represent mean \pm standard deviation, $n \geq 6$. Asterisks indicate significant differences compared with untreated samples based on Student's $t$ test; *, **, and $* * *$ indicate $P<0.05,0.01$, and 0.001 , respectively. Three independent experiments gave similar results. 
reported two trends for NPQ: a decrease and an increase that cancel each other and, hence, rendered NPQ unsuitable for distinction of infected and noninfected tissues. Here, shortterm treatment with flg22 led to a decrease in NPQ. On the other hand, virulent pathogens, which successfully suppress
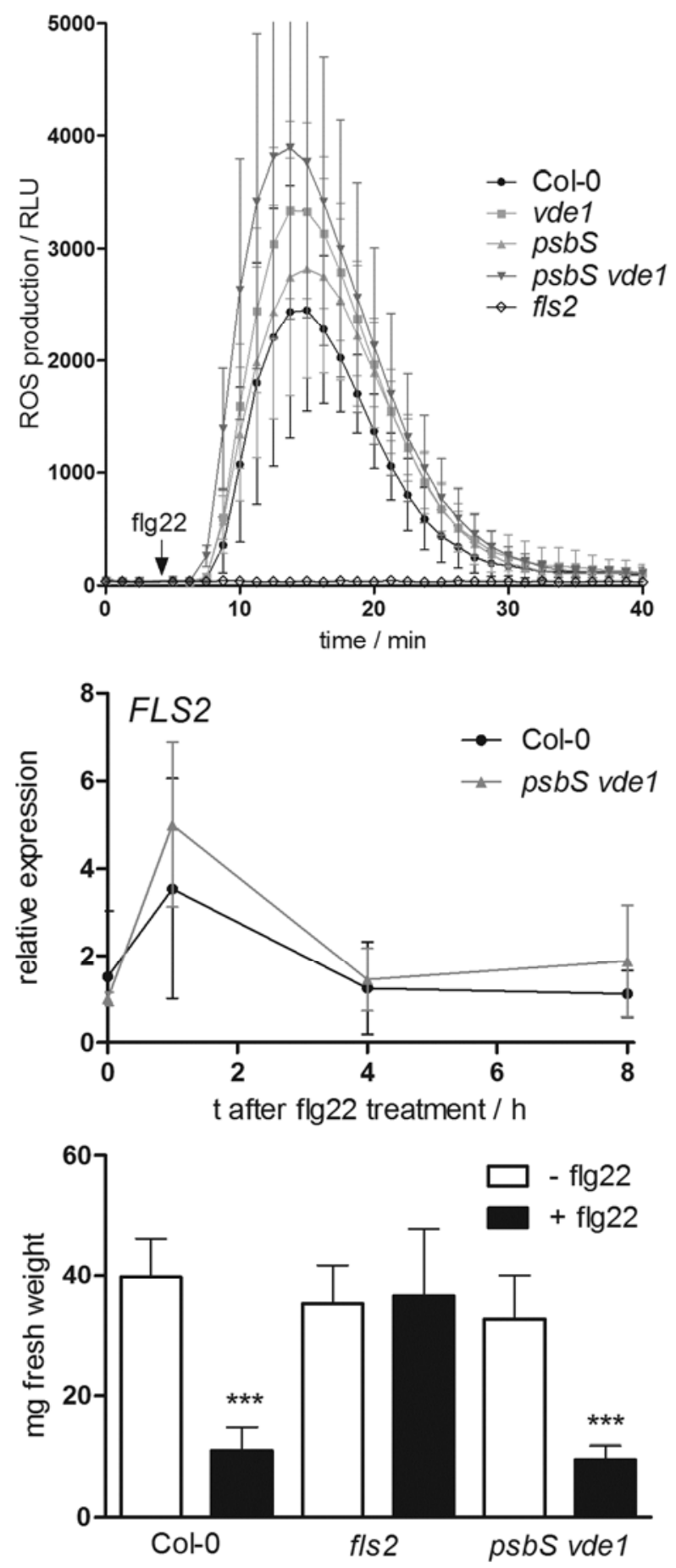

PTI, would cause an increase in NPQ, leading to the second dynamic trend during infection. Further studies of NPQ and its molecular components in infections with nonhost, virulent, and avirulent pathogens under identical growth conditions will shed light on the underlying mechanisms.
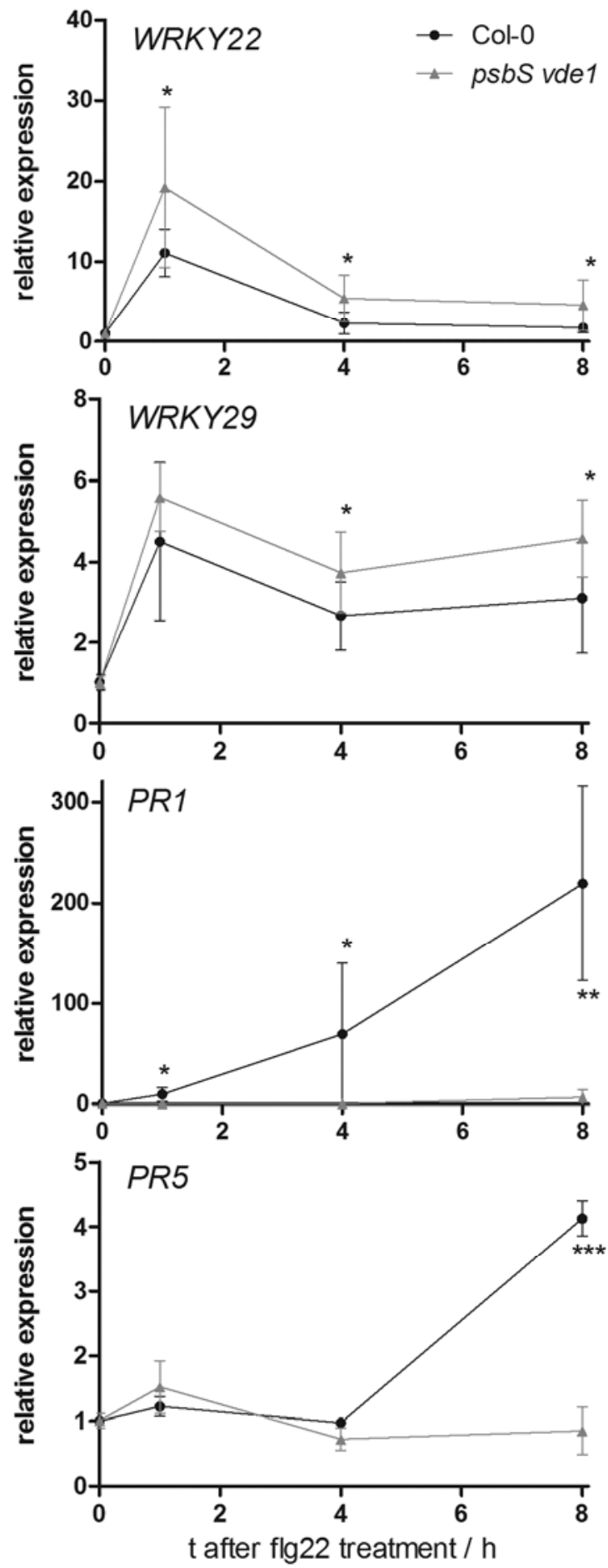

Fig. 5. The $p s b S v d e 1$ mutant has altered defense responses. Several flg22-triggered responses were analyzed in $p s b S$, $v d e 1$, and $p s b S v d e 1$. The oxidative burst, measured for $50 \mathrm{~min}$ in leaf disks, is enhanced in the single mutants and even more in the double mutant. FLS2 and defense gene expression was measured by quantitative reverse-transcriptase polymerase chain reaction in 2 -week-old seedlings treated with $10 \mu \mathrm{M}$ flg 22 for the indicated time. Expression was normalized to actin levels and to untreated conditions. Despite unaltered $F L S 2$ expression, the $p s b S v d e 1$ mutation influences defense gene expression: induction of early-induced transcription factors WRKY22 and WRKY29 is enhanced, while induction of the late-induced genes PRI and PR5 is completely abolished. To measure the growth inhibition, seedlings of the indicated mutants were grown for 7 days in the absence or presence of flg22. Whereas $f l s 2$ is insensitive to flg22, $p s b S$ vde 1 responds to the same extent as Col-0 to flg22. Values represent mean \pm standard deviation, $n \geq 6$. Asterisks indicate significant differences compared with untreated samples based on Student's $t$ test; *, **, and *** indicate $P<0.05,0.01$, and 0.001 , respectively. At least three independent experiments gave similar results. 
We have observed changes inside the chloroplast that occur rapidly upon PAMP perception. PAMPs are perceived outside the cell and trigger cytosolic signaling cascades. However, transmission of the signal to the chloroplast via the two envelop membranes is, to date, not understood. For the observed changes in NPQ upon flg22 treatment, which serves as the main model for PAMP perception in this study, the FLS2/BAK1 receptor system is required; changes caused by elf18 or chitin required the respective receptors EFR and CERK1 (Fig. 2), but downstream components are, as yet, largely unidentified. Obvious candidates are known signaling pathways involving MAPK cascades or CDPK, where the signal from different PAMPs might already converge to signal through a common pathway to the chloroplast. Flg22 induces accumulation of the plant hormone salicylic acid (SA) (Tsuda et al. 2008b), whose biosynthesis occurs partly in the chloroplast. It is unlikely that SA accumulation causes the changes in photosynthetic parameters, because SA accumulation in response to flg 22 treatment occurs later ( 3 to $6 \mathrm{~h}$ ) (Tsuda et al. 2008a) than changes in NPQ (20 min to $1 \mathrm{~h}$ ). However, proteins involved in flg22-triggered accumulation of SA such as SA induction deficient (SID2), which is localized in the chloroplast, or phytoalexin deficient (PAD4) (Tsuda et al. 2008a), might transmit the signal to NPQ. PAD4 is part of a regulatory hub controlling resistance to pathogens, which comprises, in addition, enhanced disease susceptibility (EDS1), a positive regulator of basal immunity, and a senescence-associated protein (SAG101) (Wiermer et al. 2005). EDS1, even though it is not localized in chloroplasts, is involved in processing of chloroplastic ROS (Straus et al. 2010). Thereby, this node integrates signals from light stress and immunity (Mühlenbock et al. 2008) and might play a role in fls 22 signaling to the chloroplast. In addition to hormonal signals, second messengers such as $\mathrm{Ca}^{2+}$ might transmit the signal to the chloroplast and serve as a signal integration point for the different PAMPs (Ranf et al. 2011). Upon flg22 treatment, cytosolic $\mathrm{Ca}^{2+}$ levels rise quickly (Blume et al. 2000). The chloroplast-localized $\mathrm{Ca}^{2+}$ sensing receptor CAS links cytosolic and chloroplastic $\mathrm{Ca}^{2+}$ levels (Nomura et al. 2008) and, at least in Chlamydomonas reinhardtii, is required for NPQ induction in response to changing light conditions (Petroutsos et al. 2011).

\section{NPQ modulates PTI.}

NPQ is an essential process to protect plants against excess light energy and resulting damage from formation of ROS (Müller et al. 2001). PsbS is a central player involved in NPQ (Niyogi et al. 2005) and controls the macro-organization of photosystem II complexes (Kereiche et al. 2009). In addition to increased photosensitivity, deletion of PsbS in the npq4-1 mutant alters jasmonate metabolism and responses and renders the mutant plants less attractive for herbivores (Frenkel et al. 2009). Because NPQ is affected by PTI, we tested the converse, whether mutation of NPQ has a role in PTI. Indeed, in the $p s b S$ vde1 mutant lacking NPQ, flg22-triggered ROS production at the plasma membrane and expression of early defense marker genes, the $W R K Y$ transcription factors, are enhanced. This suggests that NPQ might play a role in positively regulating PTI. Surprisingly, in contrast to apoplastic ROS and expression of $W R K Y$ genes, flg22-triggered induction of the late marker genes $P R I$ and $P R 5$ is completely abolished in the $p s b S$ vdel mutant, contradicting the role of NPQ as a positive regulator of PTI. PRI and $P R 5$ are induced by SA which, in turn, is influenced by jasmonate level (Reymond and Farmer 1998). Hence, the altered hormone balance in the $n p q 4$ mutant (Frenkel et al. 2009) could explain the opposite behavior of the different defense genes.

We propose that regulation of NPQ is an active part of the early defense program. Perception of PAMPs leads to a de- crease in energy dissipation which, in turn, could enhance the level of ROS produced by photosynthesis in the chloroplast. The resulting changes in the redox balance might prime the chloroplast so that, in case effectors are perceived in the cell, the chloroplastic ROS production can be triggered rapidly, leading to programmed cell death and ETI (Zurbriggen et al. 2009). At the same time, changes during PTI are kept marginal, so that energy production can be sustained in the presence of PAMPs when PTI is sufficient to stop the infection.

\section{MATERIALS AND METHODS}

Metabolic labeling of cell cultures.

Full metabolic ${ }^{15} \mathrm{~N} /{ }^{14} \mathrm{~N}$ labeling of the green Arabidopsis thaliana ecotype Landsberg erecta cell culture was obtained using $\mathrm{K}^{15} \mathrm{NO}_{3}$ as the sole nitrogen source in the conventional JPL growth medium (Jouanneau and Péaud-Lenoël 1967). After three passages of 7 days in ${ }^{15} \mathrm{~N}$ medium, the cell culture contained $>95 \%{ }^{15} \mathrm{~N}$.

\section{Flg22 treatment and chloroplast enrichment.}

Arabidopsis suspension cultures (Felix et al. 1999) were treated for $30 \mathrm{~min}$ or $2 \mathrm{~h}$ with $10 \mu \mathrm{M}$ flg22. ${ }^{14} \mathrm{~N}$ - and ${ }^{15} \mathrm{~N}$-labeled cells cultured with and without treatment were mixed prior to chloroplast enrichment. For each time point, we used two biological replicates that were treated independently $\left({ }^{14} \mathrm{~N}-\right.$ flg22 and ${ }^{15} \mathrm{~N}+\mathrm{flg} 22$ ). Two additional biological replicates were treated reciprocally $\left({ }^{14} \mathrm{~N}+\right.$ flg 22 and ${ }^{15} \mathrm{~N}-$ flg22). Cultures of both untreated or both treated samples were mixed as controls to account for differences between the cultures.

This setup was repeated twice for each time point, resulting in eight biological replicates each. Cells were broken manually in isotonic buffer ( $0.3 \mathrm{M}$ sorbitol, $10 \mathrm{mM}$ Tricine [pH 7.8], and $5 \mathrm{mM} \mathrm{MgCl}$ ) in a homogenizer and filtered through four layers of Micracloth, and chloroplasts were collected by lowspeed centrifugation. The pellets were directly resuspended in protein extraction buffer (1\% sodium dodecyl sulfate [SDS], 1 mM EDTA, and $100 \mathrm{mM}$ Tris, $\mathrm{pH}$ 6.8) and separated by SDSpolyacrylamide gel electrophoresis.

\section{Mass spectrometry.}

Proteins in gel slices were subjected to overnight trypsin digestion after reduction and alkylation. Peptides were eluted from the gel slices, lyophilized, and stored dry at $-20^{\circ} \mathrm{C}$ until use. Peptides were resuspended in $2 \%$ acetonitrile with $0.5 \%$ formic acid for analysis by LC-tandem mass spectrometry (MS/MS).

The LC system was a nano-Acquity (Waters Corp., Milford, MA, U.S.A.) with solvents; $0.1 \%$ formic acid in water (A) and acetonitrile (B). Peptides were trapped and washed on a vented trap column (Waters Symmetry $5-\mu \mathrm{m}$ beads, 0.180 by $20 \mathrm{~mm}$ ) with $5 \% \mathrm{~B}$ at $0.015 \mathrm{ml} / \mathrm{min}$. The reverse-phase analytical separation used Waters BEH $1301.7-\mu \mathrm{m}$ beads (column $=0.075 \mathrm{bu}$ $250 \mathrm{~mm}$ ) and a flow rate of $250 \mathrm{nl} / \mathrm{min}$ with a gradient of 5 to $40 \% \mathrm{~B}$ in $95 \mathrm{~min}$. A Proxeon nanosource was used with $2 \mathrm{kV}$ applied on $10 \mu \mathrm{m}$ fused silica spraying emitter with a liquid junction (three-way PEEK union with platinum wire).

The mass spectrometer (Orbitrap XL; Thermo Scientific, Sugarland, TX, U.S.A.) used a data-dependent acquisition program of six events: one high-resolution scan over 3,002,000 $\mathrm{m} / \mathrm{z}$ at 60,000 resolution, followed by five data-dependent collision induced decay events at low resolution in the linear trap quadropole. For the high-resolution data. a lock mass of $445.120025 \mathrm{~m} / z$ was used (Olsen et al. 2005). For MS/MS events, a minimum signal of 500 ion counts was required to select the parent ion, an isolation width of $2 \mathrm{Da}$ was used, and a normalized collision energy of $35 \%$ was applied. The prescan 
option was enabled, and charge state recognition selected for $2+, 3+$, and $4+$ ions. The chromatography function was used (threshold 1,000 ion counts, peak width $10 \mathrm{~s}$, correlation 0.9). After an MS/MS event, a dynamic exclusion was applied to that $\mathrm{m} / \mathrm{z}$ for $60 \mathrm{~s}$ (over the range -0.5 to $+1.5 \mathrm{~m} / \mathrm{z}$ ).

Data were analyzed with Mascot Distiller (version 2.3.2) and default settings were used, except where specified below Peaks lists, in mascot generic format, were searched using Mascot (v 2.2) against the Arabidopsis TAIR 9 dataset, with common contaminants (keratins and trypsin) added to give a total number of sequences $(33,596 ; 13,487,679$ residues). Two tryptic miscleavages were allowed, with carboxymethylation as a fixed modification and oxidation and phosphorylation (Ser and Thr) as variable modifications. A precursor mass error of $20 \mathrm{ppm}$ was allowed, and $0.5 \mathrm{Da}$ on the fragment masses. The search result import options were a significance threshold of $<0.05$ and required bold-red. The Distiller quantitation options were correlation factor $=0.9$, standard error threshold $=0.2$, fraction threshold $=0.4$, protein ratio type was average, no normalization, peptide threshold at least homology, the incorporation of ${ }^{15} \mathrm{~N}$ was $90 \%$ (called corrections for impurity), simple ratios were used, and the extracted ion chromatogram threshold was 0.1 .

\section{Plant material and growth conditions.}

A. thaliana seedlings Col-0, fls2 (Zipfel et al. 2004), bak1-4 (Chinchilla et al. 2007), rbohD (Mersmann et al. 2010), and psbS vdel (from P. Jahns, Heinrich-Heine University, Düsseldorf, Germany) were grown under sterile conditions on plates containing Murashige and Skoog medium supplied with Nitchvitamins and $1 \%$ sucrose (MSN) for 7 days and transferred to liquid MSN for an additional 7 days.

\section{qRT-PCR.}

Total RNA was isolated from seedlings treated with flg22 for the indicated times using a plant RNeasy mini kit (Qiagen). Total RNA $(1 \mu \mathrm{g})$ was reverse transcribed into cDNA with random hexamers (Invitrogen. Carlsbad, CA, U.S.A.) using SuperScript II Reverse Transcriptase (Invitrogen). qPCR was carried out on 1:100 dilutions of the cDNA using the Brilliant II SYBR Green QPCR Master Mix containing ROX (Agilent Technologies, Santa Clara, CA, U.S.A.) on an Mx3000P RealTime PCR System (Stratagene, La Jolla, CA, U.S.A.). The expression of the photosynthetic genes $R B C L$ (5'-GGC AGC ATT CCG AGT AAC TCC TCA-3', 5'-ATT CAG CAG CTA CCG CAG CCC-3'), PsbS (5'-TTG TGT GAA GGT CCA TTG TTT GGG T-3', 5'-CCC AAC TGT GCC AAT CTT CCT ACG A-3'), ATPA (5'-TGC CCC AGG TAT TAT TTC GAG ACG T-3', 5'TTC TCG CTG GCC GCG TC- $3^{\prime}$ ), and LHCB4 (5'-ATG TTT GGC CCA TGA CAT TA-3', 5'-GGC CAA TCT GTA TAT GCG ACA-3') and the defense genes FLS2 (5'-TCT AGA CCT GAA GAT CGA CC-3', 5'-TAC GTC CAA AGT ATT CAA CC-3'), WRKY22 (5'-CAT CCG ATC AAC AGA CGA GTA AAT-3', 5'-AAA TTC GTC GGC TGA AGT CAC-3'), WRKY29 (5'-TCC TAT GAT CCC ATC CGC TG-3', 5'-CGC TTG GTG CGT ACT CGT T-3'), PRl (5'-TTC TTC CCT CGA AAG CTC AA-3', 5'-AAG GCC CAC CAG AGT GTA TG-3'), and PR5 (5'-TGC TGC CGT GGA GCT AAC GA-3', 5'-GGC GTC AGG GCA AGC GTT CT-3') was normalized to endogenous TIP41-like gene At4g34270 (Czechowski et al. 2005) and to the untreated control. Three biological replicates with three technical replicates each were measured. Statistical analysis was done using a Student's $t$ test.

\section{Chlorophyll fluorescence measurements.}

Chlorophyll fluorescence measurements were carried out using a FluorCam800 (Photon Systems Instruments, Brno,
Czech Republic). Seedlings were grown on plates for 1 week and transferred to liquid for an additional week. Prior to measurement, the medium was exchanged for water and the plants were incubated overnight. PAMPs were added to the medium to a final concentration of $10 \mu \mathrm{M}$ flg22 and elf 18 or $100 \mu \mathrm{g}$ of chitin per milliliter for $1 \mathrm{~h}$, unless otherwise indicated. Each measurement started with 10 min of dark adaptation followed the program "act1" in the Fluorcam 7 software (Photon Systems Instruments). In brief, dark adaptation and measurement at the beginning allows quantification of maximal photosystem II capacity QY ${ }^{\max }$ and adaptation to actinic light interrupted by saturating measurement flashes allows calculation of operating parameter NPQ and QY. Statistical analysis was done using a Student's $t$ test.

\section{Stomatal aperture measurements.}

Seedlings were grown on plates for 1 week and transferred to liquid for an additional week. Prior to measurement, the medium was exchanged for water and the plants were incubated overnight. Flg22 was added to a final concentration of $10 \mu \mathrm{M}$ for $1 \mathrm{~h}$. Stomata were imaged using a Nikon Eclipse Ti-U inverse fluorescence microscope. A stomatal aperture of 100 stomata for each condition was determined as the ratio between width and length (Mersmann et al. 2010) using ImageJ software.

\section{ROS and seedling growth inhibition.}

Production of extracellular ROS and the seedling growth inhibition were measured as described previously (GomezGomez et al. 1999). Luminescence was measured in a Mithras LB 940 Multimode Reader luminometer (Berthold Technologies, Bad Wildbad, Germany). Statistical analysis was done using a Student's $t$ test.

\section{ACKNOWLEDGMENTS}

We thank P. Jahns (Düsseldorf) for material and support with chlorophyll fluorescence measurements, D. MacLean (Norwich) for bioinformatics support, and E. Vollmeister (Düsseldorf) for critically reading the manuscript. This work was supported by the Gatsby Charity Foundation (to V. Göhre, A. M. E. Jones, J. Sklenář, and S. Robatzek), a postdoctoral fellowship of the DFG International Research Training Group 1525 iGRADplant (to V. Göhre), and the German Research Council (grant IRTG 1525 to A. P. M. Weber).

\section{LITERATURE CITED}

Afroz, A., Khan, M. R., Ahsan, N., and Komatsu, S. 2009. Comparative proteomic analysis of bacterial wilt susceptible and resistant tomato cultivars. Peptides 30:1600-1607.

Asai, T., Tena, G., Plotnikova, J., Willmann, M. R., Chiu, W. L., GomezGomez, L., Boller, T., Ausubel, F. M., and Sheen, J. 2002. MAP kinase signalling cascade in Arabidopsis innate immunity. Nature 415:977983.

Aslam, S. N., Erbs, G., Morrissey, K. L., Newman, M. A., Chinchilla, D., Boller, T., Molinaro, A., Jackson, R. W., and Cooper, R. M. 2009. Microbe-associated molecular pattern (MAMP) signatures, synergy, size and charge: Influences on perception or mobility and host defence responses. Mol. Plant Pathol. 10:375-387.

Barriuso, J., Solano, B. R., and Gutierrez Manero. F. J. 2008. Protection against pathogen and salt stress by four plant growth-promoting rhizobacteria isolated from Pinus sp. on Arabidopsis thaliana. Phytopathology 98:666-672.

Berger, S., Papadopoulos, M., Schreiber, U., Kaiser, W., and Roitsch, T. 2004. Complex regulation of gene expression, photosynthesis and sugar levels by pathogen infection in tomato. Physiol. Plant. 122:419-428.

Berger, S., Benediktyova, Z., Matous, K., Bonfig, K., Mueller, M. J., Nedbal, L., and Roitsch, T. 2007a. Visualization of dynamics of plant-pathogen interaction by novel combination of chlorophyll fluorescence imaging and statistical analysis: Differential effects of virulent and avirulent strains of P. syringae and of oxylipins on A. thaliana. J. Exp. Bot. 58:797-806.

Berger, S., Sinha, A. K., and Roitsch, T. 2007b. Plant physiology meets 
phytopathology: Plant primary metabolism and plant-pathogen interactions. J. Exp. Bot. 58:4019-4026.

Blume, B., Nürnberger, T., Nass, N., and Scheel, D. 2000. Receptor-mediated increase in cytoplasmic free calcium required for activation of pathogen defense in parsley. Plant Cell 12:1425-1440.

Bolton, M. D. 2009. Primary metabolism and plant defense-fuel for the fire. Mol. Plant-Microbe Interact. 22:487-497.

Bonfig, K. B., Schreiber, U., Gabler, A., Roitsch, T., and Berger, S. 2006. Infection with virulent and avirulent $P$. syringae strains differentially affects photosynthesis and sink metabolism in Arabidopsis leaves. Planta 225:1-12.

Bosco, C. D., Lezhneva, L., Biehl, A., Leister, D., Strotmann, H., Wanner, G., and Meurer, J. 2004. Inactivation of the chloroplast ATP synthase \{gamma\} subunit results in high non-photochemical fluorescence quenching and altered nuclear gene expression in Arabidopsis thaliana. J. Biol. Chem. 279:1060-1069.

Breitling, R., Armengaud, P., Amtmann, A., and Herzyk, P. 2004. Rank products: A simple, yet powerful, new method to detect differentially regulated genes in replicated microarray experiments. FEBS (Fed. Eur. Biochem. Soc.) Lett. 573:83-92.

Cellar, N. A., Kuppannan, K., Langhorst, M. L., Ni, W., Xu, P., and Young, S. A. 2008. Cross species applicability of abundant protein depletion columns for ribulose-1,5-bisphosphate carboxylase/oxygenase. J. Chromatogr. B Anal. Technol. Biomed. Life Sci. 861:29-39.

Chen, L.-Q., Hou, B.-H., Lalonde, S., Takanaga, H., Hartung, M. L., Qu, X.-Q., Guo, W.-J., Kim, J.-G., Underwood, W., Chaudhuri, B., Chermak, D., Antony, G., White, F. F., Somerville, S. C., Mudgett, M. B., and Frommer, W. B. 2010. Sugar transporters for intercellular exchange and nutrition of pathogens. Nature 468:527-532.

Chinchilla, D., Bauer, Z., Regenass, M., Boller, T., and Felix, G. 2006. The Arabidopsis receptor kinase FLS2 binds flg22 and determines the specificity of flagellin perception. Plant Cell 18:465-476.

Chinchilla, D., Zipfel, C., Robatzek, S., Kemmerling, B., Nurnberger, T., Jones, J. D., Felix, G., and Boller, T. 2007. A flagellin-induced complex of the receptor FLS2 and BAK1 initiates plant defence. Nature 448:497-500

Czechowski, T., Stitt, M., Altmann, T., Udvardi, M. K., and Scheible, W. R. 2005. Genome-wide identification and testing of superior reference genes for transcript normalization in Arabidopsis. Plant Physiol. 139:517.

Denoux, C., Galletti, R., Mammarella, N., Gopalan, S., Werck, D., De Lorenzo, G., Ferrari, S., Ausubel, F. M., and Dewdney, J. 2008. Activation of defense response pathways by OGs and Flg22 elicitors in Arabidopsis seedlings. Mol. Plant 1:423-445

Diaz-Vivancos, P., Clemente-Moreno, M. J., Rubio, M., Olmos, E., Garcia, J. A., Martinez-Gomez, P., and Hernandez, J. A. 2008. Alteration in the chloroplastic metabolism leads to ROS accumulation in pea plants in response to plum pox virus. J. Exp. Bot. 59:2147-2160

Felix, G., Duran, J. D., Volko, S., and Boller, T. 1999. Plants have a sensitive perception system for the most conserved domain of bacterial flagellin. Plant J. 18:265-276.

Ferro, M., Brugiere, S., Salvi, D., Seigneurin-Berny, D., Court, M., Moyet, L., Ramus, C., Miras, S., Mellal, M., Le Gall, S., Kieffer-Jaquinod, S., Bruley, C., Garin, J., Joyard, J., Masselon, C., and Rolland, N. 2010. AT_CHLORO, a comprehensive chloroplast proteome database with subplastidial localization and curated information on envelope proteins. Mol. Cell Proteomics 9:1063-1084.

Freeman, B. C., and Beattie, G. A. 2009. Bacterial growth restriction during host resistance to Pseudomonas syringae is associated with leaf water loss and localized cessation of vascular activity in Arabidopsis thaliana. Mol. Plant-Microbe Interact. 22:857-867.

Frenkel, M., Kulheim, C., Jankanpaa, H. J., Skogstrom, O., Dall'Osto, L., Agren, J., Bassi, R., Moritz, T., Moen, J., and Jansson, S. 2009. Improper excess light energy dissipation in Arabidopsis results in a metabolic reprogramming. BMC Plant Biol. 9:12.

Gomez-Gomez, L., and Boller, T. 2000. FLS2: An LRR receptor-like kinase involved in the perception of the bacterial elicitor flagellin in Arabidopsis. Mol. Cell 5:1003-1011.

Gomez-Gomez, L., Felix, G., and Boller, T. 1999. A single locus determines sensitivity to bacterial flagellin in Arabidopsis thaliana. Plant J. $18: 277-284$

Hammerschmidt, R. 1999. PHYTOALEXINS: What have we learned after 60 years? Annu. Rev. Phytopathol. 37:285-306.

Ishiga, Y., Uppalapati, S. R., Ishiga, T., Elavarthi, S., Martin, B., and Bender, C. L. 2009. The phytotoxin coronatine induces light-dependent reactive oxygen species in tomato seedlings. New Phytol. 181:147-160.

Ji, X., Gai, Y., Zheng, C., and Mu, Z. 2009. Comparative proteomic analysis provides new insights into mulberry dwarf responses in mulberry (Morus alba L.). Proteomics 9:5328-5339.

Jones, J. D. G., and Dangl, J. L. 2006. The plant immune system. Nature
444:323-329.

Jouanneau, J. P., and Péaud-Lenoël, C. 1967. Growth and synthesis of proteins in cell suspensions of a kinetin dependent tobacco. Physiol. Plant. 20:834-850

Kereiche, S., Kiss, A. Z., Kouril, R., Boekema, E. J., and Horton, P. 2009. The PsbS protein controls the macro-organisation of photosystem II complexes in the grana membranes of higher plant chloroplasts. FEBS (Fed. Eur. Biochem. Soc.) Lett. 584:759-764.

Kunze, G., Zipfel, C., Robatzek, S., Niehaus, K., Boller, T., and Felix, G. 2004. The $\mathrm{N}$ terminus of bacterial elongation factor Tu elicits innate immunity in Arabidopsis plants. Plant Cell 16:3496-3507.

Li, X.-P., Bjorkman, O., Shih, C., Grossman, A. R., Rosenquist, M., Jansson, S., and Niyogi, K. K. 2000. A pigment-binding protein essential for regulation of photosynthetic light harvesting. Nature 403:391-395.

Marsh, E., Alvarez, S., Hicks, L. M., Barbazuk, W. B., Qiu, W., Kovacs, L., and Schachtman, D. 2010. Changes in protein abundance during powdery mildew infection of leaf tissues of Cabernet Sauvignon grapevine (Vitis vinifera $\mathrm{L}$.). Proteomics 10:2057-2064.

Matous, K., Benediktyova, Z., Berger, S., Roitsch, T., and Nedbal, L. 2006. Case study of combinatorial imaging: What protocol and what chlorophyll fluorescence image to use when visualizing infection of Arabidopsis thaliana by Pseudomonas syringae? Photosynth. Res. 90:243-253.

Melotto, M., Underwood, W., Koczan, J., Nomura, K., and He, S. Y. 2006. Plant stomata function in innate immunity against bacterial invasion. Cell 126:969-980.

Mersmann, S., Bourdais, G., Rietz, S., and Robatzek, S. 2010. Ethylene signalling regulates accumulation of the FLS2 receptor and is required for the oxidative burst contributing to plant immunity. Plant Physiol. 154:391-400.

Mühlenbock, P., Szechynska-Hebda, M., Plaszczyca, M., Baudo, M., Mateo, A., Mullineaux, P. M., Parker, J. E., Karpinska, B., and Karpinski, S. 2008. Chloroplast signaling and LESION SIMULATING DISEASE1 regulate crosstalk between light acclimation and immunity in Arabidopsis. Plant Cell 20:2339-2356.

Müller, P., Li, X.-P., and Niyogi, K. K. 2001. Non-photochemical quenching. a response to excess light energy. Plant Physiol. 125:1558-1566.

Navarro, L., Bari, R., Achard, P., Lisón, P., Nemri, A., Harberd, N. P., and Jones, J. D. G. 2008. DELLAs control plant immune responses by modulating the balance of jasmonic acid and salicylic acid signaling. Curr. Biol. 18:650-655.

Niyogi, K. K., Grossman, A. R., and Bjorkman, O. 1998. Arabidopsis mutants define a central role for the xanthophyll cycle in the regulation of photosynthetic energy conversion. Plant Cell 10:1121-1134.

Niyogi, K. K., Li, X. P., Rosenberg, V., and Jung, H. S. 2005. Is PsbS the site of non-photochemical quenching in photosynthesis? J. Exp. Bot. $56: 375-382$.

Nomura, H., Komori, T., Kobori, M., Nakahira, Y., and Shiina, T. 2008. Evidence for chloroplast control of external $\mathrm{Ca}^{2+}$-induced cytosolic $\mathrm{Ca}^{2+}$ transients and stomatal closure. Plant J. 53:988-998.

Olsen, J. V., de Godoy, L. M., Li, G., Macek, B., Mortensen, P., Pesch, R., Makarov, A., Lange, O., Horning, S., and Mann, M. 2005. Parts per million mass accuracy on an Orbitrap mass spectrometer via lock mass injection into a C-trap. Mol. Cell. Proteomics 4:2010-2021.

Petroutsos, D., Busch, A., Janßen, I., Trompelt, K., Bergner, S. V., Weinl, S., Holtkamp, M., Karst, U., Kudla, J., and Hippler, M. 2011. The chloroplast calcium sensor CAS is required for photoacclimation in Chlamydomonas reinhardtii. Plant Cell 23:2950-2963.

Pineda, M., Sajnani, C., and Baron, M. 2010. Changes induced by the Pepper mild mottle tobamovirus on the chloroplast proteome of Nicotiana benthamiana. Photosynth. Res. 103:31-45.

Ranf, S., Eschen-Lippold, L., Pecher, P., Lee, J., and Scheel, D. 2011. Interplay between calcium signalling and early signalling elements during defence responses to microbe- or damage-associated molecular patterns. Plant J. 68:100-113.

Reymond, P., and Farmer, E. E. 1998. Jasmonate and salicylate as global signals for defense gene expression. Curr. Opin. Plant Biol. 1:404411.

Rodriguez-Moreno, L., Pineda, M., Soukupova, J., Macho, A. P., Beuzon, C. R., Baron, M., and Ramos, C. 2008. Early detection of bean infection by Pseudomonas syringae in asymptomatic leaf areas using chlorophyll fluorescence imaging. Photosynth. Res. 96:27-35.

Straus, M. R., Rietz, S., Ver Loren van Themaat, E., Bartsch, M., and Parker, J. E. 2010. Salicylic acid antagonism of EDS1-driven cell death is important for immune and oxidative stress responses in Arabidopsis. Plant J. 62:628-640.

Swarbrick, P. J., Schulze-Lefert, P., and Scholes, J. D. 2006. Metabolic consequences of susceptibility and resistance (race-specific and broadspectrum) in barley leaves challenged with powdery mildew. Plant Cell Environ. 29:1061-1076. 
Torres, M. A., Dangl, J. L., and Jones, J. D. G. 2002. Arabidopsis gp91 ${ }^{\text {phox }}$ homologues AtrbohD and AtrbohF are required for accumulation of reactive oxygen intermediates in the plant defense response. Proc. Natl. Acad. Sci. U.S.A. 99:517-522.

Tsuda, K., Glazebrook, J., and Katagiri, F. 2008a. The interplay between MAMP and SA signaling. Plant Signal. Behav. 3:359-361.

Tsuda, K., Sato, M., Glazebrook, J., Cohen, J. D., and Katagiri, F. 2008b. Interplay between MAMP-triggered and SA-mediated defense responses. Plant J. 53:763-775.

Wan, J., Zhang, X. C., and Stacey, G. 2008. Chitin signaling and plant disease resistance. Plant Signal. Behav. 3:831-833.

Wiermer, M., Feys, B. J., and Parker, J. E. 2005. Plant immunity: The EDS1 regulatory node. Curr. Opin. Plant Biol. 8:383-389.

Yang, H., Huang, Y., Zhi, H., and Yu, D. 2010. Proteomics-based analysis of novel genes involved in response toward soybean mosaic virus infection. Mol. Biol. Rep. 38:511-521.

Zhou, W., Eudes, F., and Laroche, A. 2006. Identification of differentially regulated proteins in response to a compatible interaction between the pathogen Fusarium graminearum and its host, Triticum aestivum. Proteomics 6:4599-4609.

Zipfel, C., Robatzek, S., Navarro, L., Oakeley, E. J., Jones, J. D., Felix, G., and Boller, T. 2004. Bacterial disease resistance in Arabidopsis through flagellin perception. Nature 428:764-767.

Zurbriggen, M. D., Carrillo, N., Tognetti, V. B., Melzer, M., Peisker, M., Hause, B., and Hajirezaei, M. R. 2009. Chloroplast-generated reactive oxygen species play a major role in localized cell death during the nonhost interaction between tobacco and Xanthomonas campestris pv. vesicatoria. Plant J. 60:962-973. 